Research Article

\title{
Are we Ready for Controlling Community Transmission of COVID 19 in India?
}

\author{
Vidya Jha', Dinesh TA², Prem Nair
}

\begin{abstract}
${ }^{1}$ Assistant Professor, Asst. Medical Administrator, Amrita Institute of Medical Sciences and Research Center, Amrita Vishwa Vidyapeetham University, Cochin, Kerala, India.

${ }^{2}$ Professor \& HOD, Master of Hospital Administration, Amrita Institute of Medical Sciences and Research Center, Amrita Vishwa Vidyapeetham University, Cochin, Kerala, India.

${ }^{3}$ Professor, Department of Hospital Administration, Amrita Institute of Medical Sciences and Research Center, Amrita Vishwa Vidyapeetham University, Cochin, Kerala, India.
\end{abstract}

DOI: https://doi.org/10.24321/2455.7048.202003

\section{I $\quad \mathbf{N} \quad \mathbf{F} \quad \mathbf{O}$}

\author{
Corresponding Author: \\ Dr. Vidya Jha, Asst. Medical Administrator, Amrita \\ Institute of Medical Sciences and Research Center, \\ Amrita Vishwa Vidyapeetham University, Cochin, \\ Kerala, India. \\ E-mail Id: \\ vidyajha29@gmail.com \\ Orcid Id: \\ https://orcid.org/0000-0001-7524-3566 \\ How to cite this article: \\ Jha V, Dinesh TA, Nair P. Are we Ready for \\ Controlling Community Transmission of COVID \\ 19 in India? Epidem Int 2020; 5(1): 10-13.
}

Date of Submission: 2020-03-22

Date of Acceptance: 2020-03-28

\section{$\begin{array}{llllllll}\text { A } & \text { B } & \mathbf{S} & \mathbf{T} & \mathbf{R} & \mathbf{A} & \mathbf{C} & \mathbf{T}\end{array}$}

\begin{abstract}
The globe is witnessing COVID-19 pandemic and in India the confirmed cases are increasing day by day. Impact of limits on diagnostic testing in Indian population (1.38 billion) and on controlling the COVID-19 outbreak will be huge. These issues can be mitigated up to a certain limit by increased availability of diagnostic tests or the use of a point-ofcare diagnostic test. The buzz word is "Social distancing" and over 63\% of the population should practice it to make it effective. If appropriate measures are not taken, all hospital beds will be occupied in the coming 1-2 months and there will be shortage of Personal protective equipment and masks for healthcare workers. Are we doing enough to protect our healthcare workers? Are we ready to control community transmission of COVID-19 in India?
\end{abstract}

Keywords: COVID-19, Diagnostic Tests, Social Distancing, Community Transmission
The latest updates on COVID-19 cases in India suggest that the virus is everywhere and it's time to accept to move into a new phase in our response strategy. As of March 27 $7^{\text {th }}$, there were 863 cases in India and growing and 20 deaths, meaning you're probably going to see the numbers increasing soon. ${ }^{1}$ The numbers also reflect that the underlying burden of illness is much larger than what is being reported and the virus has been circulating for a few weeks in India. The center and state officials did a commendable job in closing schools, colleges, malls, parks, mandate travel restrictions across borders, or quarantine travelers coming into the state/ country at an appropriate time.

The criticality in creating and approving diagnostic tests for the corona virus that lets healthcare professionals know where the virus is and how far it's spreading can be understood by looking how South Korea (population $51.47 \mathrm{~m}$ ) has done so far. It has run more than 330,000 corona virus tests (8652 confirmed cases, 94 deaths). ${ }^{2}$ It is the most expansive and well-organized testing program in the world, in combination with extensive efforts to isolate infected people and trace and quarantine their contacts. In comparison, at the emergence of corona cases in USA not only were the Centers for Disease Control and Prevention Covid-19 test kits flawed, but few people qualified for Covid-19 testing. It is for sure that shortages in testing for Corona virus will put people at risk. 


\section{Impact of Limits on Diagnostic Testing in Indian Population ( 1.38 billion) and on Controlling the Outbreak}

- Waiting time for testing: Some people who have the virus may put others at risk while they are waiting to be tested. Higher the waiting time, more will be the chances. One infected person generally infects 2-3 others and which will further spread exponentially.

- $\quad$ Limiting diagnostic tests can lead to the (preventable) spread of the virus and expose health care workers to unnecessary risk.

- There are chances of outbreaks being missed as health officials are incapable for looking for them. That's what happened in Iran. Recently it is reported in Bhilwara, Rajasthan that out of 19 positive cases, 15 are medical practitioners. ${ }^{3}$

- Some people may have abdominal pain before respiratory symptoms - which goes unscreened. The entire spectrum of COVID-19 is yet unknown. Few reports of atypical presentation like abdominal pain, diarrhea, dizziness and anorexia have been published in JAMA. ${ }^{4}$ This means cases that do not present in the classic way with fever and respiratory symptoms are being missed.

These issues can be mitigated up to a certain limit by increased availability of diagnostic tests or the use of a point-of-care diagnostic test. This will help healthcare professionals to perform tests on the spot and diagnose patients with the disease. The Food and Drug Administration has approved the first rapid point-of-care COVID-19 test that can deliver results in less than an hour ( $\sim 5 \mathrm{~min}$ ) on $22^{\text {nd }}$ March 2020. ${ }^{5}$ According to Cepheid (a Silicon Valley diagnostics company) officials, the test kits will be made available by the end of March.

Recently $\left(21^{\text {st }}\right.$ March), the Indian government has expanded testing for the illness and it now can be tested in private laboratory which has NABL accreditation for real-time PCR assay for RNA virus. ${ }^{6}$ The increasing number of confirmed cases in India can be attributed to this fact.

With a population of 1.38 billion, and increasing COVID-19 cases India desperately needs a point-of-care diagnostic test to reach out to its people. Not just for the public but also for healthcare workers who will be enabled to make quick decisions and isolate patients.

In India, one cannot deny the fact that majority of people might not get tested due to unaffordable test price. All attempts should be made to make these tests available at a reasonable price or even free.

\section{The three public health tools that could (possibly) slow the spread of the outbreak include}

- Isolation is separating confirmed infections and sick from other healthy people, so that they can get better without infecting anyone else. (E.g. 2003 SARS outbreak was contained by isolation.)

- Quarantine is restricting the movement of, or isolating, people who might have been exposed to an infection but who aren't yet sick.

- $\quad$ Social Distancing (SD) refers to a slew of tactics meant to keep people from congregating in large crowds, to slow the spread of a virus.

By this time, we know that all of these might be called for during an outbreak just like in COVID-19 to varying degrees of effectiveness. Delhi's case No. 10 is an apt example of a chain reaction where six people linked to her have tested positive for COVID-19. However, isolation cannot totally stop the outbreak as too many undiagnosed cases or those with mild symptoms are already out there who may be spreading the virus.

With isolation, contact tracing is important. As in the Delhi's case No. 10, district health officials have put all those people who came in contact with the lady and other 6 under surveillance.

In a quarantine, all suspected people with chances of exposure to an infection are asked to stay at home or another place i.e. isolated from other people.

But what if the virus is circulating broadly in a population? That's where social distancing measures come in. It aims to keep people around six feet apart from each other.

Quarantine and isolation deal with specific individuals but social distancing applies to whole communities/ population.

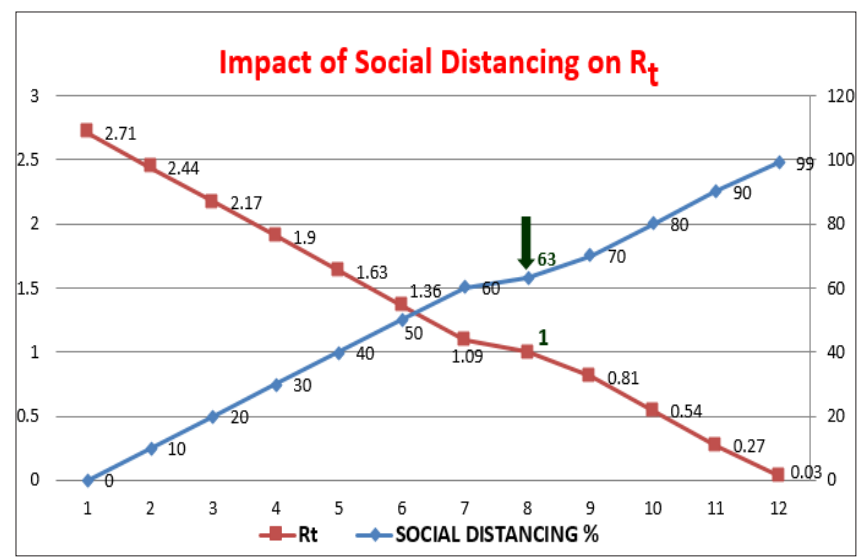

Figure I.Impact of social distancing on reproduction rate

It is only at $63 \%$ reduction in social contact that the time it takes for the COVID-19 outbreak to double, to 2229.42 days from 6.00 days, with a $R t$ of 1.00 . When $R_{0}>1$, it means that a disease will grow. In other words, for stopping the spread we need Social Distancing $>63 \%$ (Figure 1 ).

Public containment measures implemented in recent days will take a week or longer to have an effect. It is important 
to understand that with an incubation period of 2 to 7 days and a waiting time for test results of 2 to 3 days, new cases reflect infections that was incurred from more than a week ago. ${ }^{7}$ When containment fails, the exponential growth of cases can transform a public health emergency into an operational crisis.

Severe COVID-19 cases require a mean of approximately 13 days of respiratory support in a hospital set-up. ${ }^{7}$ Such lengthy treatment time will further stress resources. This time in the hospital system multiplied by the arrival rate (using Little's law) can give us a modeling system capacity before a queue develops. In case of a large Indian hospital with 100 beds in the intensive care unit, assume that at any given time, $75 \%$ of these beds are occupied. The remaining 25 beds are the effective extra capacity to absorb patients with COVID-19. Because of the long treatment time (approximately 13 days), the number of new patients that can be accommodated per day during an extended outbreak is low. Admitting more than 1.92 patients per day ( 25 beds divided by 13 days) with respiratory failure will lead to an unstable system and queues. With as many as $15 \%$ of documented infections resulting in severe disease, the situation in India may soon be tough to handle.

Hospital administrators, in India need to start preparing for worst case scenarios now. The planning will require a multidisciplinary effort from physicians, nurses, respiratory therapists, pharmacists, supply chain managers and volunteers.

Some countries like South Korea and Taiwan have been successful at combating and mitigating COVID-19 through aggressive testing and containment measures with least mortality. Their success has been mostly attributed to massive testing, adaptive policy recommendations for different regions, and communication by public officials that is constant and transparent.

As of March 27 (2pm), about 863 cases of Covid-19 had been diagnosed in India. ${ }^{1}$ The number of infected cases has increased over the last 4 days probably by increase in the number of testing labs. Given the substantial underdiagnosis at present due to limitations in testing for the corona virus and asymptomatic patients, let's say there are 2,000 current cases, a conservative starting bet.

We can expect a doubling of cases every six days, according to epidemiological studies. With ramping up of diagnostic capabilities, we may expect the confirmed COVID-19 cases to rise faster in the short term as but this is how fast we can expect actual new cases to rise in the absence of substantial mitigation measures.

That means we are looking at about 64,000 Indian cases by the end of April; $2,56,000$ by May $15^{\text {th }} ; 10,24,000$ by May $31^{\text {st; }}$, and so on.

\section{What does a Case Load of this Size Mean for Indian Health Care System?}

Using data from National Health Profile-2019, there are $7,13,986$ total government hospital beds available in India. This amounts to 0.55 beds per 1000 population.

(U.S. has about 2.8 hospital beds per 1,000 people, South Korea and Japan have more than 12 hospital beds per 1,000 people and China has 4.3 per 1,000). For a population of 1.38 billion, there are about 7,13,986 government hospital beds. At any given time, about $75 \%$ of them are occupied. That leaves about 1,78,497 beds available nationwide.

The majority of people with Covid-19 can be managed at home. But among 44,000 cases in China, about $15 \%$ required hospitalization and $5 \%$ ended up in critical care. In Italy, the statistics so far are even more dismal: More than half of infected individuals require hospitalization and about $10 \%$ need treatment in the ICU. Let us assume that $\sim 10 \%$ of cases warrant hospitalization as Indian population has high rates of chronic conditions like cardiovascular disease and diabetes, which are also associated with the severity of Covid-19.

At a $10 \%$ hospitalization rate, all hospital beds in the India will be filled by the $1^{\text {st }}$ week of June. And with many patients requiring weeks of care, turnover will slow to a crawl as beds fill with Covid-19 patients. If $20 \%$ of cases require hospitalization, we run out of hospital beds by about May $31^{\text {st }}$.

But this presumes there is no increase in demand for beds from other non-Covid-19 causes. With the health care system becoming increasingly burdened and prescription medication shortages start to appear (due to disruption in supply chain), people with chronic conditions may find themselves slipping into states of medical distress requiring hospitalization and even intensive care.

Registered medical practitioners in India are about 800,000. As Covid-19 cases saturate nearly every state, virtually all health care workers will be expected to wear masks. Assuming if only 600,000 (excluding other healthcare professionals) of them are working on any given day they would quickly run out the $\mathrm{N} 95$ respirators within two days if each worker only got one mask per day.

The global healthcare system is facing huge shortage of personal protective equipment PPE including masks, N95 respirators and even gowns. India is no different from other countries and this shortage will in turn cripple the health professional's ability to respond to the COVID-19 pandemic. As the doubling rate of virus is reducing and going by the Italy and USA stats, India is also headed towards a high case burden and huge shortage of masks and PPE.

At a time when India and other countries are desperately 
trying to "flatten the curve" by reducing new cases, reusing masks, gowns and eye shields by health workers while attending to many patients will be a significant factor in spreading COVID-19. There are over 6000 healthcare professionals ( $7.7 \%$ of total cases) in Italy and over 3400 cases ( $\sim \%$ of total cases) in China, over 3475 cases ( $6 \%$ of total cases) in Spain reported to have contracted corona virus. $^{1}$

It would be great if we are able to produce these masks, locally and nationally to keep pace with this level of demand, especially since most of the states will be simultaneously experiencing the same kind of shortages.

Another critical point in hospital management and operations is that shortages of these two resources (masks and beds) will occur simultaneously and will have a grave impact on each other's severity. Even with full Personal Protective Equipment (PPE), health care professionals are becoming infected while treating patients with Covid-19. If masks become scarce or doctors and nurses become infected, it will lead to profound shortage of staff that further will aggravate the situation. The same applies to other medical equipment and devices like ventilators. Therefore, the burning question right now is are we doing enough to protect our healthcare workers? Are they prepared to fill in the gaps when country needs them at the crisis hour?

Even if the risk of death for healthy individuals is very low, but the fraction of COVID cases that are severe makes it different from the other respiratory diseases. It is the duty of every individual to understand the magnitude of this global disease. Social distancing and hand washing are the bare minimum which is the need of the hour in order to slow the rise in cases so that health care systems are less overwhelmed.

Are we ready for the situation where healthcare needs exceed the system's capacity of India anytime? If not then we are headed for a significant loss of life. We should learn lessons from Italy and Wuhan. The COVID-19 pandemic is going to inevitably spread further. We need large scale manufacturing of PPE and masks with an intact supply chain to distribute to every hospital in India.

\section{The Best Time to Act is RIGHT NOW!}

P.S Even if some of the assumptions made by the authors, like the fraction of severe cases or the number of current cases, are off even by several-fold, it changes the overall timeline only by days or weeks.

\section{Conflicts of Interest: None \\ References}

1. https://www.worldometers.info/coronavirus/\# countries.
2. https://www.wired.co.uk/article/uk-coronavirusspread-app-phone-data.

3. https://www.indiatoday.in/india/story/rajasthan-18coronavirus-patients-including-15-doctors-bhilwaracontinues-battle-covid-19-1660064-2020-03-26.

4. Wang $\mathrm{D}, \mathrm{Bo} \mathrm{Hu}$, Chang Hu, Fangfang Zhu, Xing Liu et al. Clinical Characteristics of 138 Hospitalized Patients With 2019 Novel Coronavirus-Infected Pneumonia in Wuhan, China. JAMA.2020;323(11):1061-1069. doi:10.1001/jama.2020.1585.

5. https://www.fda.gov/news-events/pressannouncements/coronavirus-covid-19-update-fdaissues-first-emergency-use-authorization-point-carediagnostic.

6. https://www.mohfw.gov.in/pdf/Notificationofl CMguidelinesforCOVID19testinginprivatelaboratoriesil ndia.pdf.

7. Guan WJ, Ni ZY, Hu Y et al. China Medical Treatment Expert Group for Covid-19. Clinical characteristics of coronavirus disease 2019 in China. N Engl J Med. 2020. doi:10.1056/NEJMoa2002032. 